\title{
Correlation functions and momentum distribution of one-dimensional Bose systems
}

\author{
G.E. Astrakharchik and S. Giorgini \\ ${ }^{1}$ Dipartimento di Fisica, Università di Trento, and BEC-INFM, I-38050 Povo, Italy
}

(October 25, 2018)

\begin{abstract}
The ground-state correlation properties of a one-dimensional Bose system described by the LiebLiniger Hamiltonian are investigated by using exact quantum Monte Carlo techniques. The pair distribution function, static structure factor, one-body density matrix and momentum distribution of a homogeneous system are calculated for different values of the gas parameter ranging from the Tonks-Girardeau to the mean-field regime. Results for the momentum distribution of a harmonically trapped gas in configurations relevant to experiments are also presented.
\end{abstract}

PACS numbers: 03.75.Fi, 05.30.Fk, 67.40.Db

The recent experimental achievements in realizing quasi one-dimensional (1D) quantum degenerate Bose gases confined in harmonic traps [1-3], have attracted a great interest on the ground-state properties of these systems. The 1D regime is reached in highly anisotropic traps, where the axial motion of the atoms is weakly confined while the radial motion is frozen to zero point oscillations by the tight transverse confinement. In these conditions and if the characteristic range of interparticle interactions is much smaller than the typical length of the transverse confinement, the system can be described by the Lieb-Liniger model of $\delta$-interacting $1 \mathrm{D}$ bosons. For a homogeneous system the Hamiltonian has the form

$$
H_{L L}=-\frac{\hbar^{2}}{2 m} \sum_{i=1}^{N} \frac{\partial^{2}}{\partial z_{i}^{2}}+g_{1 D} \sum_{i<j} \delta\left(z_{i}-z_{j}\right),
$$

where $m$ is the atomic mass and $g_{1 D}=2 \hbar^{2} / m\left|a_{1 D}\right|$ is the coupling constant in terms of the 1D effective scattering length $a_{1 D}$ [4]. Recent numerical simulations using exact quantum Monte Carlo techniques have explicitly shown the cross-over from the three-dimensional (3D) to the 1D Lieb-Liniger regime for the energetics and structural properties of trapped systems $[5,6]$. The Hamiltonian $H_{L L}$ is expected to provide the correct description also for the correlation properties of a quasi 1D system. In this Letter we calculate for the first time, using exact quantum Monte Carlo methods, the pair distribution function and the one-body density matrix of a Lieb-Liniger gas in its ground state. By taking the Fourier transform of the one- and two-body correlation function we obtain results for the momentum distribution and the static structure factor of the system respectively. These correlation functions can be accessed in experiments using ballistic expansion and Bragg spectroscopy techniques and can provide a signature of the exotic properties of these quantum degenerate 1D systems.

The equation of state and excitation spectrum of a homogeneous system of bosons described by the Hamiltonian (1) have been calculated exactly in the thermodynamic limit in Ref. [7] for any value of the dimensionless gas parameter $n\left|a_{1 D}\right|$, where $n$ is the particle density.
If $n\left|a_{1 D}\right| \gg 1$, the system is in the weakly interacting regime and mean-field theory can be applied. In this case, the energy per particle is given by the Gross-Pitaevskii (GP) prediction: $E / N=g_{1 D} n / 2$. In the opposite limit, $n\left|a_{1 D}\right| \ll 1$, the system enters the Tonks-Girardeau (TG) regime of a gas of impenetrable bosons. In this regime, which has been first investigated by Girardeau [8], the system acquires fermionic properties in the sense that there exists an exact mapping between the wave function of the interacting bosons and the wave function of a noninteracting Fermi gas. The energy per particle coincides in this case with the Fermi energy: $E / N=\pi^{2} \hbar^{2} n^{2} /(6 m)$.

We study the following correlation functions: the onebody density matrix, which in terms of the ground-state wave function $\Psi_{0}\left(z_{1}, . ., z_{N}\right)$ is defined as

$$
g_{1}(z)=\frac{N}{n} \frac{\int \Psi_{0}^{*}\left(z_{1}+z, . ., z_{N}\right) \Psi_{0}\left(z_{1}, . ., z_{N}\right) d z_{2} . . d z_{N}}{\int\left|\Psi_{0}\left(z_{1}, . ., z_{N}\right)\right|^{2} d z_{1} . . d z_{N}},
$$

and the pair distribution function

$$
g_{2}\left(\left|z_{1}-z_{2}\right|\right)=\frac{N(N-1)}{n^{2}} \frac{\int\left|\Psi_{0}\left(z_{1}, . ., z_{N}\right)\right|^{2} d z_{3} . . d z_{N}}{\int\left|\Psi_{0}\left(z_{1}, . ., z_{N}\right)\right|^{2} d z_{1} . . d z_{N}} .
$$

By taking the Fourier transform of these correlation functions one obtains the momentum distribution $n(k)=$ $n \int d z e^{i k z} g_{1}(z)$ and the static structure factor $S(k)=$ $1+n \int d z e^{i k z}\left[g_{2}(z)-1\right]$.

For a system of impenetrable bosons $\left(n\left|a_{1 D}\right| \ll 1\right)$ the above correlation functions can be calculated analytically. By exploiting the Bose-Fermi mapping one finds the following result for the pair distribution function [8]

$$
g_{2}(z)=1-j_{0}^{2}(\pi n|z|),
$$

where $j_{0}$ is a spherical Bessel function. The corresponding static structure factor is given by

$$
S(k)=\left\{\begin{array}{cc}
|k| /(2 \pi n) & (|k|<2 \pi n) \\
1 & (|k|>2 \pi n) .
\end{array}\right.
$$


The one-body density matrix of a TG gas has also been calculated by performing analytic expansions for short and long distances (see Ref. [9] and references therein). The long-range behavior is given by $g_{1}(z) \propto 1 / \sqrt{|z|}[10]$, corresponding to an infrared divergence in the momentum distribution $n(k) \propto 1 / \sqrt{|k|}$.

Beyond the TG regime full closed-form expressions of the correlation functions are not known. The long-range asymptotics of the one-body correlation function can be obtained from the hydrodynamic theory of low-energy excitations $[11,12]$. One finds the following power-law decay

$$
g_{1}(z) \propto 1 /|z|^{\alpha}
$$

where $\alpha=m c /(2 \pi \hbar n)$ is fixed by the density $n$ and by the speed of sound $c$. The above result holds for $|z| \gg \xi$ where $\xi=\hbar /(\sqrt{2} m c)$ is the healing length of the system. The velocity of sound can be obtained from the equation of state through the compressibility of the system $m c^{2}=$ $n \partial \mu / \partial n$, where $\mu=d E / d N$ is the chemical potential. In the TG regime $\left(n\left|a_{1 D}\right| \ll 1\right)$ one finds $m c=\pi \hbar n$ and thus $\alpha=1 / 2$ as anticipated above. In the opposite GP regime $\left(n\left|a_{1 D}\right| \gg 1\right)$, the result is $\alpha=1 /\left(\pi \sqrt{2 n\left|a_{1 D}\right|}\right)$ which decreases as $n\left|a_{1 D}\right|$ increases. Of course, result (6) excludes the existence of Bose-Einstein condensation even in the ground-state [13]. The infrared behavior of the momentum distribution follows immediately from Eq. (6)

$$
n(k) \propto 1 /|k|^{1-\alpha}
$$

holding for $|k| \ll 1 / \xi$. Furthermore, the hydrodynamic theory allows one to calculate the static structure factor in the long-wavelength regime $|k| \ll 1 / \xi$. One finds the well-known result

$$
S(k) \rightarrow \hbar|k| /(2 m c)
$$

characteristic of phonon excitations. Recently, the short range behavior of the one- and two-body correlation functions has also been investigated. The value at $z=0$ of the pair correlation function can be obtained from the equation of state through the Hellmann-Feynman theorem [14]: $g_{2}(z=0)=-\left(m\left|a_{1 D}\right| / \hbar^{2}\right) n^{2} d\left[(E / N) / n^{2}\right] / d n$. The result approaches zero in the TG regime and unity in the GP regime. Furthermore, the first few terms of the short-range series expansion of the one-body correlation function $g_{1}(z)=1+\sum_{i=1}^{\infty} c_{i}|n z|^{i}$ have been calculated in Ref. [9] again exploiting the knowledge of the equation of state.

In this Letter we provide a complete calculation of the spatial dependence of the one- and two-body correlation functions for values of the gas parameter ranging from the TG to the deep GP regime. The calculation is carried out using the diffusion Monte Carlo (DMC) method, which allows one to solve exactly, apart from statistical uncertainty, the many-body Schrödinger equation for the ground state of a Bose system [15]. We consider a system of $N$ particles described by the Hamiltonian (1), with periodic boundary conditions. The system has size $L$ and linear density $n=N / L$. Importance sampling is used through the trial wave function $\psi_{T}\left(z_{1}, \ldots, z_{N}\right)=\prod_{i<j} f\left(z_{i j}\right)$, where $f(z)$ is a two-body term which we choose as

$$
f(z)=\left\{\begin{array}{cc}
A \cos [k(|z|-B)] & (|z|<\bar{Z}) \\
\sin ^{\beta}(\pi|z| / L) & (|z|>\bar{Z}) .
\end{array}\right.
$$

The $z=0$ boundary condition $f^{\prime}\left(0^{+}\right)-f^{\prime}\left(0^{-}\right)=$ $2 f(0) /\left|a_{1 D}\right|$, which accounts for the $\delta$-function potential, fixes the parameter $k$ through the condition $k\left|a_{1 D}\right| \tan (k B)=1$. The remaining parameters $A, B$ and $\beta$ are fixed by requiring that the function $f(z)$, its derivative $f^{\prime}(z)$ and the local energy $-2 f^{\prime \prime}(z) / f(z)$ be continous at the matching point $z=\bar{Z}$. The value of the matching point $\bar{Z}$ is a variational parameter that we optimize using variational Monte Carlo (VMC). For $|z|<\bar{Z}$ the Jastrow term corresponds to the exact solution of the two-body problem and is expected to provide a correct description of short-range correlations. Longrange correlations arising from phonon excitations are instead accounted for by the functional dependence of $f(z)$ for $|z|>\bar{Z}[11]$. Notice that at $|z|=L / 2$, which sets the limits of our simulation box, $f=1$ and particles are uncorrelated. The TG wave function $\Psi_{0}^{T G}=$ $\prod_{i<j}\left|\sin \left[\pi\left(z_{i}-z_{j}\right) / L\right]\right|$ is obtained as a special case of our trial function $\psi_{T}$ for $\bar{Z}=B=L / 2$ and $k L=\pi$. The optimized Jastrow function introduced above gives for all values of $n\left|a_{1 D}\right|$ variational results for the energy which are almost identical to the exact DMC ones. The level of accuracy of the trial wave function is particularly important for the calculation of $g_{1}(z)$. In fact, the pair distribution function $g_{2}(z)$ is calculated using the method of "pure" estimators, unbiased by the choice of the trial function [16]. Due to the non-local property of the onebody density matrix, the function $g_{1}(z)$ can instead be obtained only through the extrapolation technique. For an operator $A$ which does not commute with the Hamiltonian, the output of the DMC method is the mixed estimator $\left\langle\Psi_{0}|A| \psi_{T}\right\rangle$ and one can approximate the "pure" estimator by $\left\langle\Psi_{0}|A| \Psi_{0}\right\rangle=2\left\langle\Psi_{0}|A| \psi_{T}\right\rangle-\left\langle\psi_{T}|A| \psi_{T}\right\rangle$, where $\left\langle\psi_{T}|A| \psi_{T}\right\rangle$ is the variational estimator. Of course, this procedure is very accurate only if $\psi_{T} \simeq \Psi_{0}$. We find that DMC and VMC give results for $g_{1}(z)$ which are very close and we believe that the extrapolation technique is in this case exact. We also find that $g_{1}(z)$ is strongly affected by finite size effects particularly in the GP regime, where simulations of quite large systems are needed to obtain stable results. In order to control these effects we perform simulations with number of particles ranging from $N=100$ to $N=500$.

In Fig. 1 we show the results of the pair distribution function for values of $n\left|a_{1 D}\right|$ ranging from the TG to the GP regime (the corresponding values of the energy per 
particle are shown in the inset). For the smallest value of the gas parameter $\left(n\left|a_{1 D}\right|=10^{-3}\right)$ we find that $g_{2}(z)$ exactly coincides with the TG result (4). We also find that the values of $g_{2}(0)$ exactly reproduce the results obtained from the equation of state. We notice that for small values of the gas parameter correlations die out very quickly and $g_{2}(z) \simeq 1$ after few interparticle distances. For larger values of $n\left|a_{1 D}\right|$ correlations extend over much larger distances. This result is also visible from the behavior of the static structure factor (shown in Fig. 2) which in the GP regime is dominated by long-wavelength fluctuations.

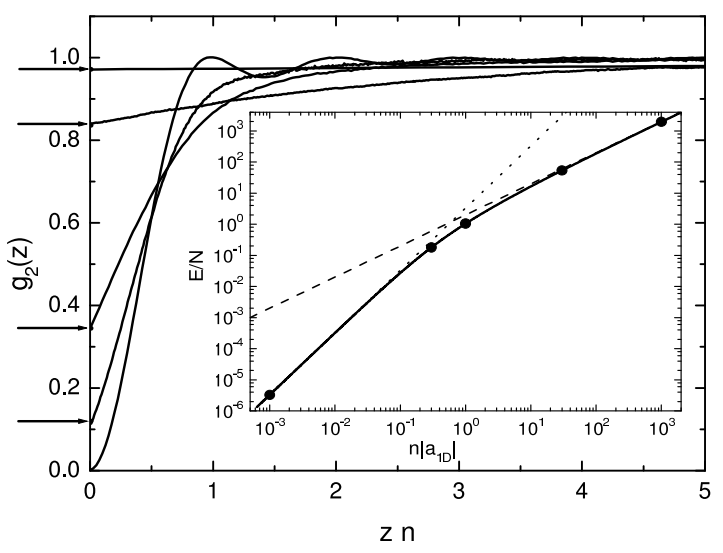

FIG. 1. Pair distribution function for different values of the gas parameter. In ascending order of the value at $z=0$ : $n\left|a_{1 D}\right|=10^{-3}, 0.3,1,30,10^{3}$. Arrows indicate the value of $g_{2}(0)$ as obtained from the equation of state. Inset: energy per particle for the same values of $n\left|a_{1 D}\right|$ (symbols), Lieb-Liniger equation of state (solid line), GP limit (dashed line), TG limit (dotted line). Energies are in units of $\hbar^{2} /\left(2 m a_{1 D}^{2}\right)$.

In Fig. 3 we present results for the one-body density matrix. We see that for distances considerably larger than the healing length $\xi, g_{1}(z)$ is well reproduced by the asymptotic behavior (6) for which we calculate the proportionality coefficient through a best fit to the DMC results. The deviations from the power law decay at the largest distances $(z \simeq L / 2)$ are due to finite size effects. In Fig. 4 we show the corresponding results for the momentum distribution. This is obtained from the Fourier transform of the calculated one-body correlation function at short distances and of the fits to the power-law decay (6) at large distances. We notice that the infrared asymptotic behavior (7) is recovered for values of $k$ considerably smaller than the inverse healing length $1 / \xi$. At large $k$ the numerical noise of our results is too large to extract evidences of the $1 / k^{4}$ behavior predicted in [9].

Let us now turn to harmonically trapped systems. In the $1 \mathrm{D}$ regime the system is described by the Hamiltonian $H_{L L}+\sum_{i=1}^{N} m \omega_{z}^{2} z_{i}^{2} / 2$ with an effective coupling constant given by $g_{1 D}=2 \hbar^{2} a /\left(m a_{\perp}^{2}\right)$, where $a$ is the $3 \mathrm{D} s$-wave scattering length and $a_{\perp}=\sqrt{\hbar /\left(m \omega_{\perp}\right)}$ is the length fixed by the tight transverse confinement $[4,6]$. Consequently, the value of the effective $1 \mathrm{D}$ scattering length is given by the ratio $\left|a_{1 D}\right|=a_{\perp}^{2} / a$. The relevant parameters are: the ratio $a / a_{\perp}$, the anisotropy parameter $\lambda=\omega_{z} / \omega_{\perp}$ and the number of trapped particles $N$.

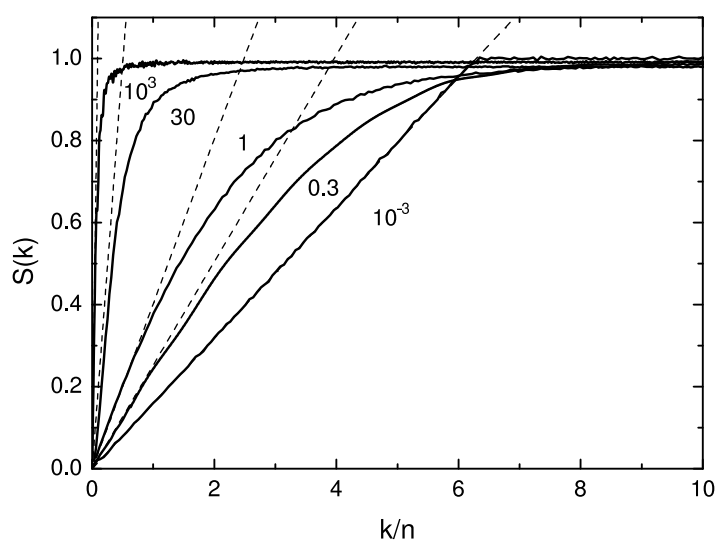

FIG. 2. Static structure factor for the same values of $n\left|a_{1 D}\right|$ as in Fig. 1 (solid lines). The dashed lines are the corresponding long-wavelength asymptotics from Eq. (8).

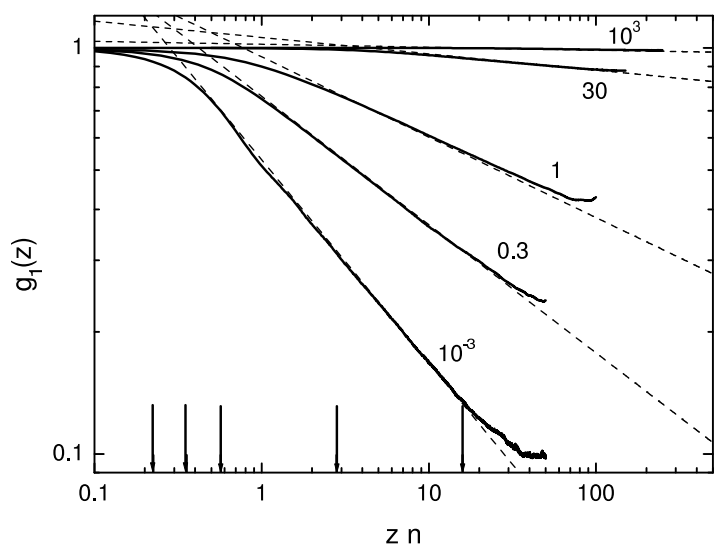

FIG. 3. One-body density matrix for the same values of $n\left|a_{1 D}\right|$ as in Fig. 1 (solid lines). The dashed lines are the fits to the corresponding long-wavelength asymptotics from Eq. (6). The arrows indicate the value of $\xi n$ : the leftmost corresponds to $n\left|a_{1 D}\right|=10^{-3}$, the rightmost to $n\left|a_{1 D}\right|=10^{3}$.

The DMC simulation is carried out using the following trial wave function for the importance sampling: $\psi_{T}\left(z_{1}, . ., z_{N}\right)=\prod_{i} g\left(z_{i}\right) \prod_{i<j} f\left(z_{i j}\right)$, where $g(z)=$ $\exp \left(-\alpha_{z} z^{2}\right)$ is a one-body term which accounts for the 
confinement in the longitudinal direction and is fixed by the variational parameter $\alpha_{z}$. In the two-body term we neglect long-range correlations, which are irrelevant in the trapped case, and we choose $f(z)$ as given by (9) for $|z|<\bar{Z}$ and $f(z)=1$ for $|z|>\bar{Z}$. We consider the following configurations: $a / a_{\perp}=0.2, \lambda=10^{-3}$ and number of particles $N=5,20,100$. In Ref. [6] we have proven that in these conditions the ground-state energy and structure of the cloud is correctly described by the Lieb-Liniger equation of state in local density approximation. The momentum distribution of a trapped system is obtained as: $n(k)=\int d Z d z^{\prime} n(Z) g_{1}\left(Z+z^{\prime} / 2, Z-z^{\prime} / 2\right) e^{i k z^{\prime}}$, where $n(z)$ is the density profile and $n\left[\left(z+z^{\prime}\right) / 2\right] g_{1}\left(z, z^{\prime}\right)=$ $N \int \Psi_{0}^{*}\left(z, . ., z_{N}\right) \Psi_{0}\left(z^{\prime}, . ., z_{N}\right) d z_{2} . . d z_{N} / \int\left|\Psi_{0}\left(z_{1}, . ., z_{N}\right)\right|^{2}$ $d z_{1} . . d z_{N}$.

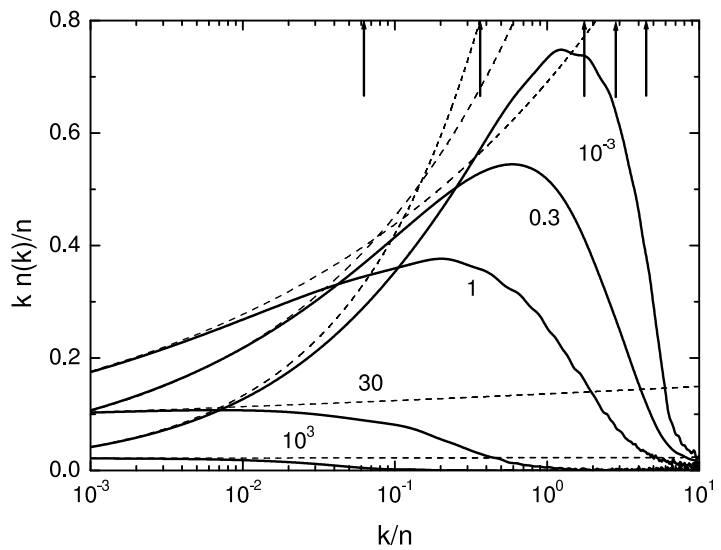

FIG. 4. Momentum distribution for the same values of $n\left|a_{1 D}\right|$ as in Fig. 1 (solid lines). The dashed lines correspond to the infrared behavior of Eq. (7). The arrows indicate the value of $1 /(\xi n)$ : the rightmost corresponds to $n\left|a_{1 D}\right|=10^{-3}$, the leftmost to $n\left|a_{1 D}\right|=10^{3}$.

In Fig. 5 we show the results for the momentum distribution. Note the peaks becoming sharper with increasing $N$. In the case of $N=5$ and $N=20$, we find that the rounding off of $n(k)$ at $k \sim 1 / R_{z}$, where $R_{z}$ is the size of the cloud in the axial direction, washes out completely the divergent behavior at small momenta. For the largest system with $N=100$ the value of the gas parameter is $n_{0}\left|a_{1 D}\right| \simeq 1.1$, where $n_{0}$ is the density in the center of the trap, and the corresponding value of the exponent $\alpha$ is $\alpha \simeq 0.19$. In the region of wave vectors $1 / R_{z}<k<1 / \xi$, where the healing length $\xi$ is estimated in the center of the trap, we find some evidence of the infrared behavior (7) (see inset of Fig. 5). In order to see a cleaner signature of this effect one should consider much larger systems.

In conclusion, we have carried out a complete study of the one- and two-body correlation functions of the 1D Lieb-Liniger model at $T=0$. We have also investigated how the presence of harmonic trapping modifies the behavior of the momentum distribution in configurations relevant to experiments.

We would like to thank J. Boronat for useful discussions. This research is supported by the Ministero dell'Istruzione, dell'Università e della Ricerca (MIUR).

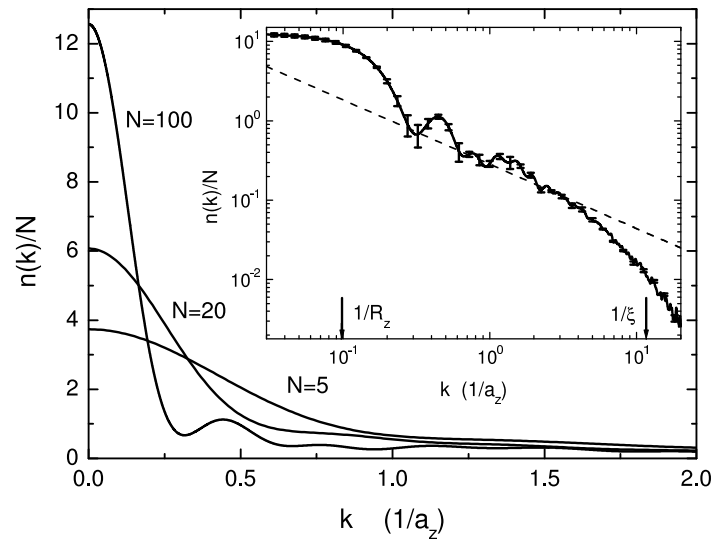

FIG. 5. Momentum distribution of a trapped system. Inset: momentum distribution for $N=100$ (solid line) on a $\log$-log scale. The dashed line is a fit to $1 / k^{1-\alpha}$ with $\alpha=0.19$. The momentum distribution is in units of $a_{z}=\sqrt{\hbar /\left(m \omega_{z}\right)}$.

[1] A. Görlitz et al., Phys. Rev. Lett. 87, 130402 (2001).

[2] F. Schreck et al., Phys. Rev. Lett. 87, 080403 (2001).

[3] M. Greiner et al., Phys. Rev. Lett. 87, 160405 (2001).

[4] M. Olshanii, Phys. Rev. Lett. 81, 938 (1998).

[5] D. Blume, Phys. Rev. A 66, 053613 (2002).

[6] G.E. Astrakharchik and S. Giorgini, Phys. Rev. A 66, 053614 (2002).

[7] E.H. Lieb and W. Liniger, Phys. Rev. 130, 1605 (1963); E.H. Lieb, ibid. 130, 1616 (1963).

[8] M. Girardeau, J. Math. Phys. (N.Y.) 1, 516 (1960).

[9] M. Olshanii and V. Dunjko, e-print cond-mat/0210629.

[10] A. Lenard, J. Math. Phys. 5, 930 (1964).

[11] L. Reatto and G.V. Chester, Phys. Rev. 155, 155 (1967).

[12] M. Schwartz, Phys. Rev. B 15, 1399 (1977); F.D.M. Haldane, Phys. Rev. Lett. 47, 1840 (1981).

[13] T.D. Schultz, J. Math. Phys. 4, 666 (1963).

[14] D.M. Gangardt and G.V. Shlyapnikov, e-print condmat/0207338.

[15] For a general reference on the DMC method see for example J. Boronat and J. Casulleras, Phys. Rev. B 49, 8920 (1994).

[16] J. Casulleras and J. Boronat, Phys. Rev. B 52, 3654 (1995). 